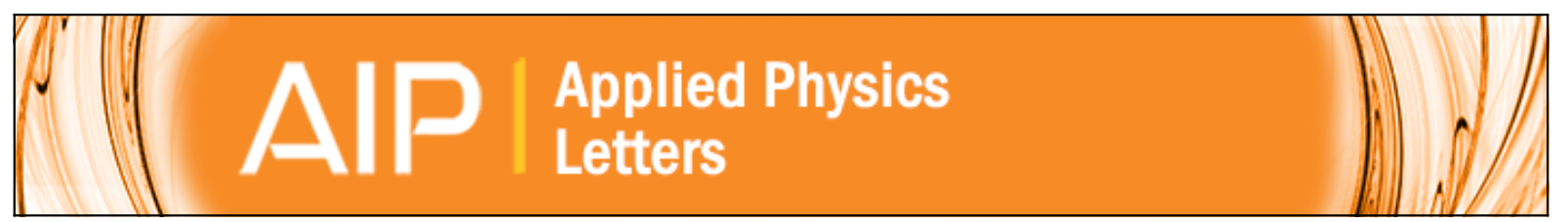

\title{
Amplified spontaneous emission of Rhodamine 6G embedded in pure deoxyribonucleic acid
}

Ileana Rau, Adam Szukalski, Lech Sznitko, Andrzej Miniewicz, Stanislaw Bartkiewicz, Francois Kajzar, Bouchta Sahraoui, and Jaroslaw Mysliwiec

Citation: Applied Physics Letters 101, 171113 (2012); doi: 10.1063/1.4764535

View online: http://dx.doi.org/10.1063/1.4764535

View Table of Contents: http://scitation.aip.org/content/aip/journal/apl/101/17?ver=pdfcov

Published by the AIP Publishing

\section{Articles you may be interested in}

Low threshold amplified spontaneous emission from dye-doped DNA biopolymer

J. Appl. Phys. 111, 113107 (2012); 10.1063/1.4728218

Effect of deoxyribonucleic acid on nonlinear optical properties of Rhodamine 6G-polyvinyl alcohol solution J. Appl. Phys. 109, 023110 (2011); 10.1063/1.3520657

Enhanced amplified spontaneous emission using layer-by-layer assembled cowpea mosaic virus J. Appl. Phys. 105, 013511 (2009); 10.1063/1.3056137

Optical detection of deoxyribonucleic acid hybridization with In Ga N/Ga N multiple quantum wells Appl. Phys. Lett. 92, 261910 (2008); 10.1063/1.2948907

Thin-film lasers based on dye-deoxyribonucleic acid-lipid complexes Appl. Phys. Lett. 81, 1372 (2002); 10.1063/1.1501164

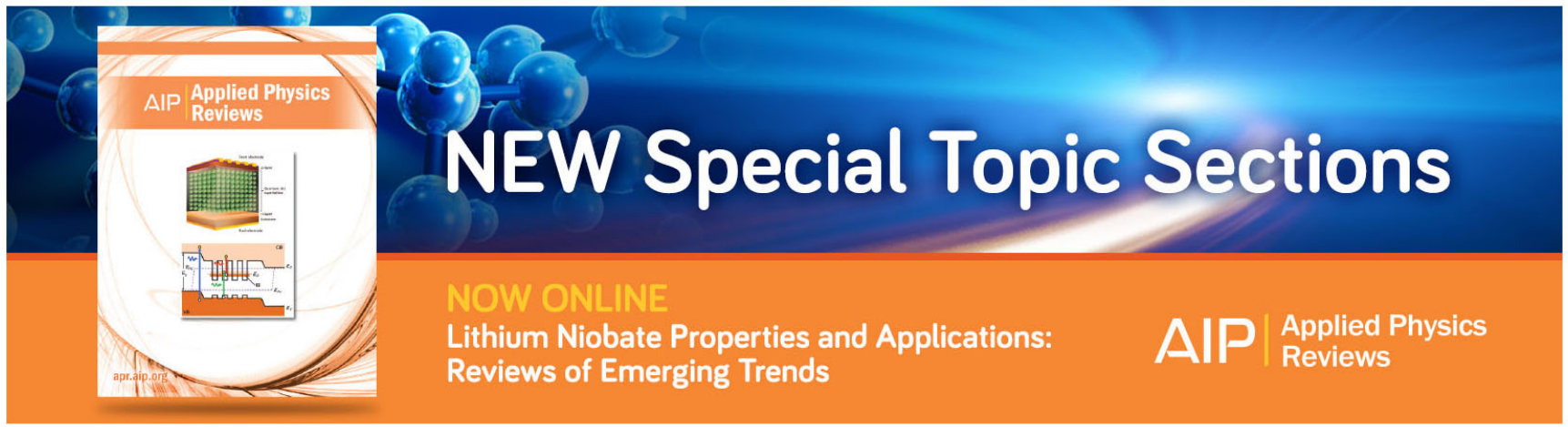




\title{
Amplified spontaneous emission of Rhodamine $6 \mathrm{G}$ embedded in pure deoxyribonucleic acid
}

\author{
Ileana Rau, ${ }^{1}$ Adam Szukalski, ${ }^{2}$ Lech Sznitko, ${ }^{2}$ Andrzej Miniewicz, ${ }^{2}$ Stanislaw Bartkiewicz, ${ }^{2}$ \\ Francois Kajzar, ${ }^{1}$ Bouchta Sahraoui, ${ }^{3}$ and Jaroslaw Mysliwiec ${ }^{2,3, a)}$ \\ ${ }^{1}$ Politechnica University of Bucharest, STR. Polizu 1, Bucharest, Romania \\ ${ }^{2}$ Wroclaw University of Technology, Wyb. Wyspianskiego 27, 50370 Wroclaw, Poland \\ ${ }^{3}$ Lunam Universite, Universite Angers, CNRS UMR 6200, Laboratoire MOLTECH-Anjou, 2 bd Lavoisier, \\ 49045 Angers cedex, France
}

(Received 6 September 2012; accepted 15 October 2012; published online 25 October 2012)

\begin{abstract}
Deoxyribonucleic acid (DNA) is commonly viewed as a genetic information carrier. However, now it is recognized as a nanomaterial, rather than as a biological material, in the research field of nanotechnology. Here, we show that using pure DNA, doped with rhodamine 6G, we are able to observe amplified spontaneous emission (ASE) phenomenon. Moderate ASE threshold, photodegradation, and reasonable gain coefficient observed in this natural host gives some perspectives for practical applications of this system in biophotonics. Obtained results open the way and will be leading to construction of truly bio-lasers using nature made luminophores, such as anthocyanins. (C) 2012 American Institute of Physics. [http://dx.doi.org/10.1063/1.4764535]
\end{abstract}

The search of materials for luminescence, amplified spontaneous emission (ASE), and lasing is one of the important subjects of modern photonics. Such materials for photonics devices should show several properties, like photosensitivity, excellent optical quality, ease of processing, to be environmentally- and bio-friendly. Dye-doped organic systems find many applications in photonics. Interest in these systems is focused not only on investigations of their fundamental physicochemical properties (spectroscopic measurements-absorption, fluorescence excitation) but also in chemical sensing, optical switching, and in medicine (biomedical imaging techniquesblood pressure control during surgery, etc.). Unfortunately, these materials show still some limitations for applications in commercial devices and are usually characterized by a short lifetime. Bioderived and bioinspired materials are of current interest because they can provide attractive physical properties while being produced by "green" technologies and being also biodegradable. Deoxyribonucleic acid (DNA) holds a very special position in this context. It can be used as a matrix for incorporating active functional molecules enabling fabrication of some photonic devices. It can lead also to future applications in molecular photonics, e.g., in technologies where single molecules are used for storage and processing of information or for microlasing. DNA is also an extraordinarily exciting system for biolasing applications mostly due to the exceptional capability of DNA double helix strand structure to interact with various molecules (nonbinding, groove binding, and semi-intercalation) and modify their properties. It was shown also that DNA is an interesting matrix for luminophores. A large up to 17 times stronger photoluminescence from thin films of sulphorhodamine in DNA-surfactant matrix, as compared to polymethyl methacrylate (PMMA) matrix, was observed by $\mathrm{Yu}$ et al. ${ }^{1} \mathrm{DNA}$, and particularly, the complex DNA-surfactant (where as surfactant cetyltrimethyl or benzyltrimethyl ammonium chloride can be used) are known to be excellent matrices for lasing molecules. ${ }^{2}$ However, despite the

${ }^{a)}$ Electronic mail: jaroslaw.mysliwiec@pwr.wroc.pl, Tel.: +48 713202317. large number of papers reporting observation of ASE from DNA-surfactant-luminophore complexes, ${ }^{1,3-9}$ there were no reports on amplified spontaneous emission in a pure DNA without surfactant and doped with lasing dye.

In this study, we focused out our attention on a system, which contain pure DNA doped with the well known lasing molecule rhodamine 6G (DNA-Rh6G). The used in this study DNA biopolymer, extracted from water solution salmon roe, was supplied by Ogata from Chitose Institute of Science and Technology. Its initial molecular mass was reduced by sonication to ca. $5 \times 10^{5}$ daltons. The solutions used for thin film fabrication were prepared according to the following procedure. The concentration of Rh6G in water solution $(1 \mathrm{ml})$ was $10^{-4} \mathrm{~mol} / \mathrm{dm}^{3}$, and properly the mass of this dye constituted $1 \%$ weight with respect to DNA weight. At first, the compounds were weighted and dissolved in distilled water (every in $1 \mathrm{ml}$ of distilled water). After that, both solutions were carefully mixed and put into the refrigerator for one day. For the thin film preparation on glass substrate, we have used procedure described by Samoc et al. ${ }^{10}$ with the use of the drop-casting method. Films were dried in air at $21-25^{\circ} \mathrm{C}$ and about $50 \%$ relative humidity for about a day and stored in ambient air for several days.

The thickness of the layer was around $\mathrm{d} \sim 3 \mu \mathrm{m}$. First, the excitation, emissive, and absorptive properties of DNA-Rh6G thin film were determined at room temperature. Spectral measurements were carried out using a Hitachi F-4500 fluorescence spectrophotometer (range of study 350-700 nm). The spectral resolution was maintained at $1 \mathrm{~nm}$ both for excitation and emission spectra. The normalized excitation and fluorescence emission spectra of DNA-Rh6G are displayed in Fig. 1. The full width at half maximum (FWHM) of fluorescence emission band is about $39 \mathrm{~nm}$. For measurements of amplified spontaneous emission, we assembled a typical experimental set-up, which is schematically shown in Fig. 2, where a linearly polarized light beam with wavelength $\lambda=532 \mathrm{~nm}$, coming from the frequency doubled neodymium doped yttrium aluminum garnet (Nd:YAG) laser (pulse duration $8 \mathrm{~ns}$ and $11 \mathrm{~Hz}$ repetition rate), after passing through a diaphragm, was 


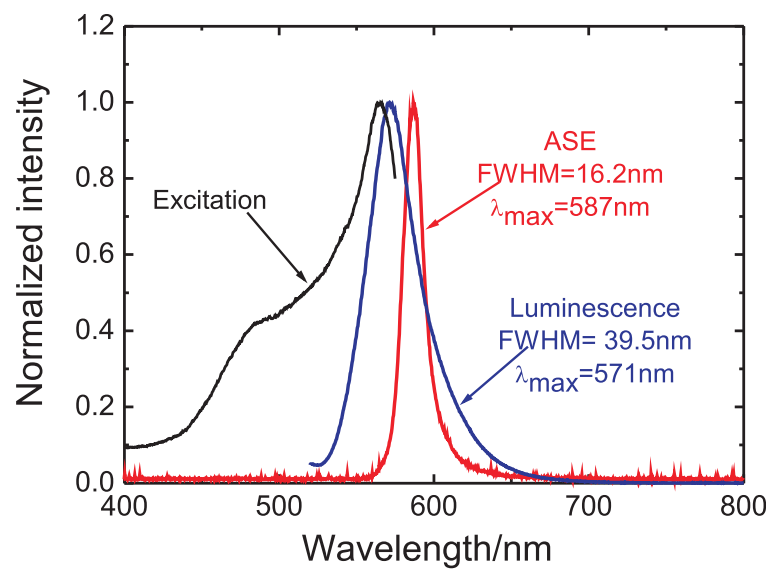

FIG. 1. Fluorescence excitation, emission, and ASE spectra of DNA-Rh6G sample at room temperature. Excitation wavelength $\lambda=532 \mathrm{~nm}$ and ASE excitation energy density $\rho=56.3 \mathrm{~mJ} / \mathrm{cm}^{2}$.

used to excite the sample. The amplified spontaneous emission, emerging from the edge of the sample, was collected by a lens and fed to optical fiber and analyzed with a fiber spectrometer (Ocean Optics), coupled to a computer. Configuration of the experiment and directionality of the emitted ASE light at suitable excitation energy let us register clear ASE signal with a little fluorescence background. For the determination of the exponential gain coefficient $\gamma$, we modified the light excitation geometry in order to get an extended stripe of the light instead of a laser spot. For that purpose, we used a converging cylindrical lens giving $\mathrm{L}=15 \mathrm{~mm}$ long and narrow stripe of light that allowed us to apply Shank method ${ }^{11}$ for evaluation of $\gamma$.

The results of amplified spontaneous emission measurements, together with excitation and emission spectra, are presented in Fig. 1. One can clearly see a characteristic narrowing of the fluorescence line width above the ASE threshold. For the reported DNA-Rh6G system, we observe variation of the luminescence bandwidth from $\Delta \lambda_{\mathrm{FWHM}} \approx 39 \mathrm{~nm}$ for the lower excitation energy density presented in Fig. 1 and measured with a conventional spectrometer, up to $\Delta \lambda_{\mathrm{FWHM}} \approx 16 \mathrm{~nm}$ for ASE light emitted from the sample and collected by the fiber spectrometer.

As one can see in Fig. 3, where the results of FWHM parameter measurements and intensity of luminescence (ASE as inset) in function of the excitation energy density are presented, the threshold of ASE appearance for DNA-Rh6G is

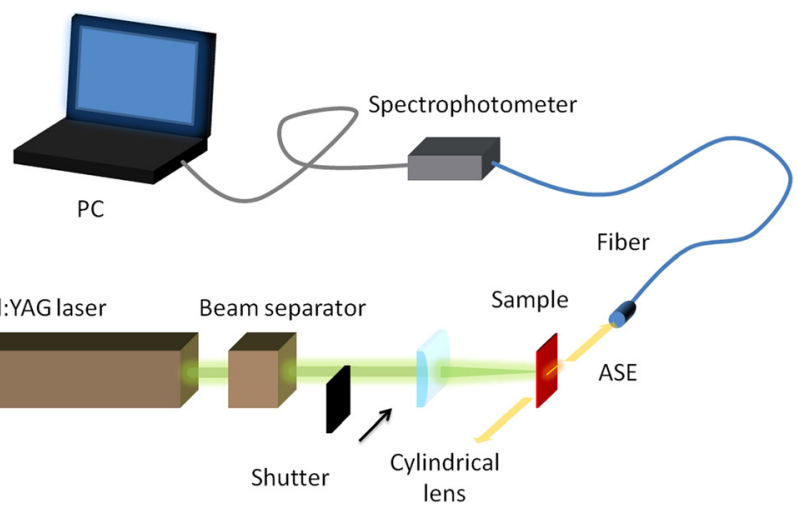

FIG. 2. Experimental set-up for ASE and gain coefficient measurements.

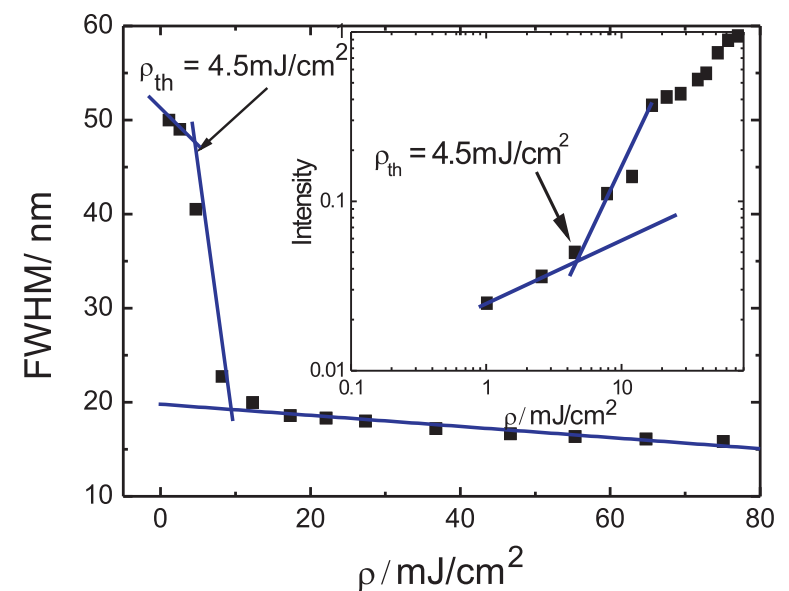

FIG. 3. Dependence of FWHM parameter (intensity of luminescence and ASE as inset) on the energy density of pumping beam for DNA-Rh6G system. $\rho_{t h}$-energy density threshold for appearance of the amplified spontaneous emission.

$\rho_{\text {th }}=4.5 \mathrm{~mJ} / \mathrm{cm}^{2}$. An important parameter of the systems showing ASE phenomenon and determining its practical applicability is the level of photodegradation. The experimental results of temporal stability of ASE signal are presented in Fig. 4. As it follows from Fig. 4, a 50\% initial value reduction of the ASE intensity occurs after around $20 \mathrm{~s}$, what is equivalent to 200 excitation light pulses. Such value is typical for most of organic dye-doped luminescent systems, what shows important role of this matrix, ${ }^{6,12-14}$ where DNA prevents the aggregation of dye molecules and traps the dye molecules in the helical structures. ${ }^{15}$

The exponential gain coefficient $\gamma$ can be determined, according to Shank method, by measuring the ASE signals at the sample edge for full length $\mathrm{L}$ and half-length $\mathrm{L} / 2$ of the light stripe. From the obtained ASE intensities $\mathrm{I}_{\mathrm{L}}$ and $\mathrm{I}_{\mathrm{L} / 2}$ for these two cases, one derives the $\gamma$ coefficient according to the equation

$$
\gamma=\frac{2}{\mathrm{~L}} \ln \left(\frac{\mathrm{I}_{\mathrm{L}}}{\mathrm{I}_{\mathrm{L} / 2}}-1\right) .
$$

Conditions of the experimental set-up like polarization state and energy density of the excitation beam where correctly selected to get the optimal ASE signal. Experimental results

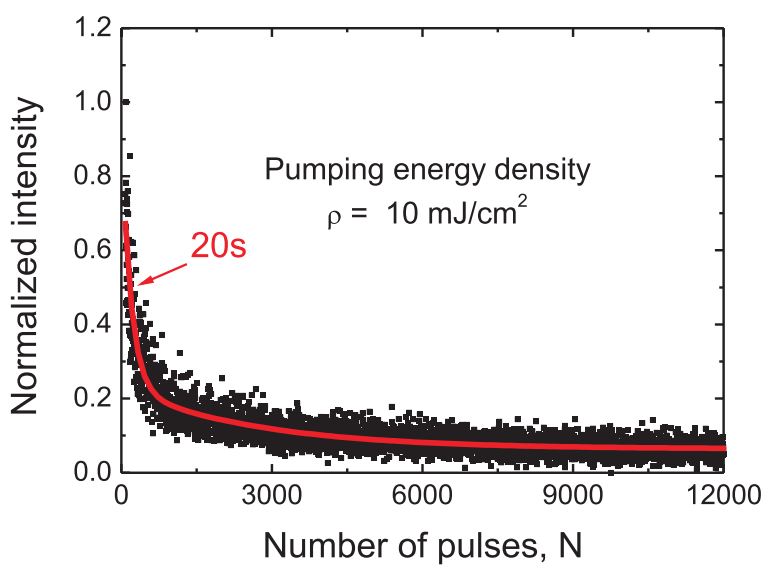

FIG. 4. Dependence of ASE intensity on number of laser pulses for DNA-Rh6G. 
and calculations based on the Eq. (1) show that average value of the exponential gain coefficient $\gamma$ in the DNA-Rh6G sample is $\gamma=6.5 \mathrm{~cm}^{-1}$.

In conclusion, the amplified spontaneous emission in a pure deoxyribonucleic acid loaded with a fluorescent dye Rhodamine 6G system was observed and characterized. The reported ASE threshold and gain coefficient $\gamma \approx 6.5 \mathrm{~cm}^{-1}$ is similar to that presented for this molecule in other organic matrices. ${ }^{6,12-14}$ Such properties gives some perspectives for practical applications of this system in biophotonics and medicine. The next step will be using nature made luminophores, such as anthocyanins, ${ }^{16}$ leading to truly biolasers.

This work was financially supported by Wroclaw University of Technology, Polish National Science Centre (Grant Nos. 2011/01/B/ST8/03317 and 2011/01/B/ST5/ 00773). J.M. and S.B. acknowledge the financial support by University of Angers and Region Pays de la Loire. I.R. and F.K. acknowledge the financial support of Romanian Ministry of Education, Research, Youth and Sports, through the UEFISCDI organisation, under Contract No. 279/ 7.10.2011, Code Project PN-II-ID-PCE-2011-3-05053. L.S. would like to acknowledge the Foundation for Polish Science for the "Start" fellowship.
${ }^{1}$ Z. Yu, J. Hagen, Y. Zhou, D. Klotzkin, J. Grote, and A. Steckl, Appl. Opt. 46(9), 1507-1513 (2007).

${ }^{2}$ Y. Kawabe and K.-I. Sakai, Nonlinear Opt. Quantum Opt. 43, 273 (2011).

${ }^{3}$ Y. Kawabe, L. Wang, S. Horinouchi, and N. Ogata, Adv. Mater. 12, 1281 (2000).

${ }^{4}$ Y. Kawabe, L. Wang, T. Nakamura, and N. Ogata, Appl. Phys. Lett. 81, 1372 (2002).

${ }^{5}$ J. Mysliwiec, L. Sznitko, S. Bartkiewicz, A. Miniewicz, Z. Essaidi, B. Sahraoui, and F. Kajzar, Appl. Phys. Lett. 94, 241106 (2009).

${ }^{6}$ J. Mysliwiec, L. Sznitko, A. Miniewicz, F. Kajzar, and B. Sahraoui, J. Phys. D: Appl. Phys. 42, 085101 (2009).

${ }^{7}$ J. Mysliwiec, L. Sznitko, A. Sobolewska, S. Bartkiewicz, and A. Miniewicz, Appl. Phys. Lett. 96, 141106 (2010).

${ }^{8}$ N. Balan, M. Hari, V. Parameswaran, and N. Nampoori, Applied Optics 48(19), 3521-3525 (2009).

${ }^{9}$ Y.-C. Hung, C.-H. Su, and H.-W. Huang, J. Appl. Phys. 111, 113107 (2012).

${ }^{10}$ A. Samoc, A. Miniewicz, M. Samoc, and J. G. Grote, J. Appl. Polym. Sci. 105, 236 (2007).

${ }^{11}$ C. V. Shank, A. Dienes, and W. T. Silfvast, Appl. Phys. Lett. 17, 307 (1970).

${ }^{12}$ G. Somasundaram and A. Ramalingam, J. Photochem. Photobiol. A 125, 93 (1999).

${ }^{13}$ A. Costela, F. Florido, I. Garcia-Moreno, R. Duchowicz, F. Amat-Guerri, J. M. Figura, and R. Sastre, Appl. Phys. B 60, 383 (1995).

${ }^{14}$ A. V. Deshpande and E. B. Namdas, Appl. Phys. B 64, 419 (1997).

${ }^{15}$ B. Nithyaja, H. Misha, P. Radhakrishnan, and V. P. N. Nampoori, J. Appl. Phys. 109, 023110 (2011).

${ }^{16}$ I. Iosub, F. Kajzar, M. Makowska-Janusik, A. Meghea, A. Tane, and I. Rau, Opt. Mater. 34, 1644 (2012). 